\title{
BOUNDED SYMMETRIC HOMOGENEOUS DOMAINS
}

\section{IN INFINITEDIMENSIONAL SPACES}

\author{
Lawrence A. Harris ${ }^{1}$
}

In this article, we exhibit a large class of Banach spaces whose open unit balls are bounded symmetric homogeneous domains. These Banach spaces, which we call $\mathrm{J}^{*}-$ algebras, are linear spaces of operators mapping one Hilbert space into another and have a kind of Jordan triple product structure. In particular, all Hilbert spaces and all $\mathrm{B}^{*}$-algebras are $\mathrm{J}^{*}$-algebras. Moreover, all four types of the classical Cartan domains and their infinite dimensional analogues are the open unit balls of $\mathrm{J}^{*}$-algebras, and the same holds for any finite or infinite product of these domains. Thus we have a setting in which a large number of bounded symmetric homogeneous domains may be studied simultaneously. A particular advantage of this setting is the interconnection which exists between function-theoretic problems and problems of functional analysis. This leads to a simplified discussion of both types of problems.

We shall see that the open unit balls of $\mathrm{J}^{*}$-algebras are natural generalizations of the open unit disc of the complex plane. In fact, we give an explicit algebraic formula for Möbius transformations of these balls and show that the origin can be mapped to any desired operator in the ball with one of the Möbius transformations. An extremal form of the Schwarz lemma then leads immediately to the representation of each biholomorphic mapping between the open unit balls of two $j^{*}$-algebras as a composition of a Möbius transformation and a linear isometry of one of the $\mathrm{J}^{*}$-algebras onto the other. Such linear isometries reduce to a multiplication by unitary operators for mappings in the identity component of the group of all biholomorphic mappings of the open unit ball of a $\mathrm{C}^{*}$-algebra with identity. However, in general, linear isometries of one $J^{*}$-algebra onto another can be complicated. Still, using the mentioned Schwarz lemma and Möbius trans" formations, we show that all such linear isometries preserve the $\mathrm{J}^{*}$-structure.

A consequence of these results is that the open unit balls of two $\mathrm{J}^{*}$-algebras are holomorphically equivalent if and only if the $\mathrm{J}^{*}$-algebras are isometrically isomorphic under a mapping preserving the $\mathrm{J}^{*}-$ structure. Another consequence is that the open unit ball of a $\mathrm{J}^{*}$-algebra is holomorphically equivalent to a product of balls if and only if the $\mathrm{J}^{*}$-algebra is isometrically isomorphic to a product of $\mathrm{J}^{*}$-algebras.

The last result connects the factorization of domains with the factorization of $\mathrm{J}^{*}$-algebras and has a number of interesting applications. For example, using Cartan's classification of bounded symmetric domains in $\mathrm{C}^{n}$, we classify all $\mathrm{J}^{*}$-algebras of dimension less than 16 . Moreover, we reduce the problem of classifying all finite dimensional $\mathrm{J}^{*}$-algebras to the problem of finding some $\mathrm{J}^{*}$-algebras whose open unit balls are holomorphically equivalent to the two exceptional Cartan domains in dimensions 16 and 27 , respectively, when such $\mathrm{J}^{*}$-malgebras exist. If there are such $\mathrm{J}^{*}$-algebras in both cases, then every bounded symmetric

1 Research partially supported by N.S.F. grant GP-33117A\#1. 
domain in $\mathbf{C}^{n}$ is holomorphically equivalent to the open unit ball of a $\mathrm{J}^{*}$-algebra. Also, for $\mathrm{J}^{*}$-algebras having an isometry, we obtain an algebraic condition which implies that the open unit ball of the $\mathrm{J}^{*}$-algebra is not holomorphically equivalent to a product of balls. As expected, none of the infinite dimensional analogues of the four types of classical Cartan domains is holomorphically equivalent to a product of balls.

The unit spheres of many $\mathrm{J}^{*}$-algebras contain small subsets playing the same role as the distinguished boundary of polydiscs. In fact, any non-empty subset of the unit sphere of a $\mathrm{J}^{*}$-algebra which is stable under the application of the Möbius transformations and under multiplication by complex numbers of unit modulus is a boundary for the algebra of all bounded complex-valued functions holomorphic in the open unit ball of the $\mathrm{J}^{*}$-algebra and continuous in its closure. Three particularly interesting stable subsets of a $\mathrm{J}^{*}$-algebra are (when non-empty) the set of extreme points of the closed unit ball, the set of all isometries, and the set of all unitary operators. For a finite dimensional $\mathrm{J}^{*}$-algebra, the Shilov boundary for the mentioned algebra of functions is the set of extreme points of the closed unit ball of the $\mathrm{J}^{*}-$ algebra. We give an algebraic characterization of extreme points and determine these explicitly for the $\mathrm{J}^{*}$-algebras whose open unit balls are classical Cartan domains.

Finally, we show that the open unit balls of a large number of $\mathrm{J}^{*}$--algebras are holomorphically equivalent to an explicitly constructed unbounded affinely homogeneous domain in the $\mathrm{J}^{*}$-algebra which plays the role of the upper half-plane. These upper half-planes are operator-theoretic analogues of Siegel domains of genus 2. Siegel's generalized upper half plane and tubes whose base is a future light cone are included as special cases.

This article generalizes and expands Chapter IV of the author's thesis, written under the direction of Professor Clifford Earle.

\section{$\S 1$. Generalities}

Let $X$ and $Y$ be complex normed linear spaces and let $O$ be an open subset of $X$. (To avoid trivialities, all normed linear spaces considered will be assumed to be different from the zero space.) A function $\mathrm{h}: \mathscr{\sigma} \rightarrow \mathrm{Y}$ is said to be holomorphic (in $\mathscr{})$ ) if the Fréchet derivative of $\mathrm{h}$ at $\mathrm{x}$ (denoted by Dh( $\mathrm{x}$ )) exists as a bounded complex-linear map of $X$ into $Y$ for each $x \in \delta$. If $\mathscr{O}^{\prime}$ is an open subset of $Y$, a function h: $\infty \rightarrow \Phi^{\prime}$ is said to be a biholomorphic mapping (of $\mathscr{\infty}$ onto $\mathscr{D}^{\prime}$ ) if the inverse function $\mathrm{h}^{-1}: \infty^{\prime} \rightarrow \infty$ exists and both $\mathrm{h}: \mathscr{\sigma} \rightarrow \mathrm{Y}$ and $\mathrm{h}^{-1}: \mathscr{O}^{\prime} \rightarrow \mathrm{X}$ are holomorphic. The sets $\mathscr{O}$ and $\mathscr{O}^{\prime}$ are said to be holomorphically equivalent if there exists a biholomorphic mapping of $\mathscr{S}$ onto $\mathcal{O}^{\prime}$. A domain $\mathscr{O}$ is said to be homogeneous if for each pair of points $x, y \in \mathscr{W}$ there exists a biholomorphic mapping h: $\mathscr{\sigma} \rightarrow \mathscr{D}$ with $h(x)=y$. If in addition the mappings $h$ can be chosen to be affine mappings, $\alpha$ is said to be affinely homogeneous. Further, $\mathscr{O}$ is said to be a symmetric domain if for each $x \in \mathscr{O}$ there exists a biholomorphic mapping $h: \sigma \rightarrow \sigma$ such that $h$ has $x$ as its only fixed point and $h^{2}=1$, where $I$ is the identity map on $\mathscr{\sigma}$.

Throughout, the open (resp., closed) unit ball of a complex normed linear space $X$ is denoted by $X_{0}$ (resp., $X_{1}$ ). Thus 


$$
X_{0}=\{x \in X:\|x\|<1\}, X_{1}=\{x \in X:\|x\| \leqslant 1\} .
$$

A point $x \in X_{1}$ is said to be an extreme point (resp., a complex extreme point) of $x_{1}$ if the only $y \in X$ satisfying $\|x+\lambda y\| \leqslant 1$ for all real (resp., complex) numbers $\lambda$ with $|\lambda| \leqslant 1$ is $y=0$. Clearly any extreme point of $X_{1}$ is a complex extreme point of $X_{1}$.

In a previous paper [14] (see also [15] and [16]), the author proved

Theorem 1. If $h: X_{0} \rightarrow Y_{0}$ is a biholomorphic mapping with $h(0)=0$, then $h$ is the restriction to $X_{0}$ of a linear isometry of $\mathrm{X}$ onto $\mathrm{Y}$.

Now if $X_{0}$ is a homogeneous domain, any biholomorphic mapping of $X_{0}$ onto $Y_{0}$ may be preceeded by a biholomorphic mapping of $X_{0}$ onto itself so that the composition takes 0 to 0 . Thus we obtain

Corollary 1. Suppose $X_{0}$ is a homogeneous domain. Then the domains $X_{0}$ and $Y_{0}$ are holomorphically equivalent if and only if the spaces $\mathrm{X}$ and $\mathrm{Y}$ are isometrically isomorphic.

For example, suppose that $H$ is a complex Hilbert space and that $H_{0}$ is holomorphically equivalent to a domain $X_{0} \times Y_{0}$. Clearly $X_{0} \times Y_{0}=(X \times Y)_{0}$ when $X \times Y$ has the norm $\|(X, y)\|=\max \|x\|$, $\left.\|y\|\right\}$, and we shall see later that $H_{0}$ is a homogeneous domain. Hence by Corollary 1 , the spaces $H$ and $X \times Y$ are isometrically isomorphic. Then since all unit vectors in $H$ are extreme points of $H_{1}$, the same must be true of $X x Y$. But for each $x \in X$ with $\|x\|=1$, the point $(x, 0)$ is a unit vector in $X x Y$ which is not an extreme point of $(X \times Y)_{1}$. Thus we conclude that $H_{0}$ is not holomorphically equivalent to any domain of the form $X_{0} \times Y_{0}$. Clearly this result contains Theorem 2.1 of [11]. (A more general result is given in Theorem 8 below.)

Further, note that if $X_{0}$ is a homogeneous domain then it is automatically symmetric. Indeed, given $x \in X_{0}$, let $g$ be a biholomorphic mapping of $X_{0}$ onto itself with $g(x)=0$, and define $h=g^{-1} \circ L_{\circ}$, where $L x=-x$ for $x \in x_{0}$. It is easy to verify that $h$ has the required properties.

It would be interesting to know whether or not Corollary 1 holds without the assumption that $x_{0}$ is a homogeneous domain.

\section{\$2. $\mathrm{J}^{*}$-algebras and Cartan domains}

Let $\mathrm{H}$ and $\mathrm{K}$ be complex Hilbert spaces and let $\mathcal{L}(\mathrm{H}, \mathrm{K})$ denote the Banach space of all bounded linear operators from $H$ to $K$ with the operator norm. For each operator $A \in \mathscr{L}(H, K)$ there is a uniquely determined operator $A^{*} \in \mathcal{L}(K, H)$ such that $(A x, y)=\left(x, A^{*} y\right)$ for all $x \in H$ and $y \in K$. It is easily verified that $*$ satisfies the usual laws of an adjoint operation $[25$, p.105] .

Definition 1. $A J^{*}$-algebra is a closed complex-linear subspace $\mathscr{I}$ of $\mathcal{L}(H, K)$ such that $A A^{*} A \in \mathcal{L}$ whenever $A \in \mathscr{U}$. 
Throughout, unless otherwise specified, $\mathfrak{U}, \mathbb{Z}$, and $\mathfrak{S}$ denote arbitrary $\mathrm{J}^{*}$-algebras.

Many familiar spaces are $\mathrm{J}^{*}$-algebras. For example, any Hilbert space $\mathrm{H}$ may be thought of as a $\mathrm{J}^{*}$ algebra since $H$ can be identified with the space $\mathcal{L}(\mathrm{C}, \mathrm{H})$, where $\mathrm{C}$ denotes the complex plane. Also any $\mathrm{C}^{*}-$ algebra is obviously a J*-algebra and hence by the Gelfand-Naimark theorem [31, p.244], any $\mathrm{B}^{*}$-algebra may be thought of as a $J^{*}$-algebra. Further, if $C$ and $D$ are self-adjoint operators in $\mathcal{L}(H)$ and $\mathcal{L}(K)$, respectively, then

$$
\mathscr{U}=\{A \in \mathcal{L}(H, K): A C=D A\}
$$

is easily seen to be $\mathrm{J}^{*}$-algebra.

Define a set $\mathfrak{U}$ to be a Cartan factor of

type $I$ if $\mathfrak{U}=\mathscr{L}(H, K)$,

type II if $\mathfrak{U}=\left\{A \in \mathscr{L}(H): A^{t}=A\right\}$,

type III if $\mathscr{U}=\left\{A \in \mathscr{L}(H): A^{t}=-A\right\}$,

where $A^{t}=Q A^{*} Q$ and $Q$ is a conjugate-linear map on $H$ with $\|Q\| \leqslant 1$ and $Q^{2}=I$. (Such a map always exists and is called a conjugation.) Polarization shows that $Q$ reverses inner products and it follows that $\left(A^{*}\right)^{t}=$ $\left(A^{t}\right)^{*}$ for all $A \in \mathcal{L}(H)$. Consequently, all the Cartan factors of type I-III are $J^{*}$-algebras. Note that the infinite dimensional domains considered in [11] and [28] are the open unit balls of Cartan factors of types I and II. Moreover, each of the Cartan domains $[10, \S 24]$ of types I-III is the open unit ball of a finite dimensional Cartan factor of the corresponding type.

A Cartan factor of type IV is a closed subspace $\mathcal{E}$ of $\mathcal{L}(\mathrm{H})$ such that the adjoint of each operator in थ is in $\mathscr{U}$ and such that the square of each operator in $\mathscr{\mathscr { U }}$ is a scalar multiple of the identity operator I on $\mathrm{H}$. It is clear from the identities

$$
\begin{aligned}
A B+B A & =(A+B)^{2}-A^{2}-B^{2} \\
A B^{*} A & =\left(A B^{*}+B^{*} A\right) A-B^{*} A^{2}
\end{aligned}
$$

that $\mathscr{Y}$ is a $\mathrm{J}^{*}$-algebra. Note that $\mathfrak{U}$ is also a Hilbert space in an equivalent norm since the equation

$$
A B^{*}+B^{*} A=2(A, B) I
$$

defines an inner product on $\mathcal{U}$ and clearly $1 / 2\|A\|^{2} \leqslant(A, A) \leqslant\|A\|^{2}$ for all $A \in \mathcal{U}$. Conversely, any Hilbert space $H$ can be obtained as the Hilbert space associated with a Cartan factor of type IV. In fact, if $x \rightarrow \bar{x}$ is a conjugation on $H$, there exists a Hilbert space $K$ and a linear map $x \rightarrow A_{X}$ of $H$ into $\mathcal{L}(K)$ such that

$$
A_{x}^{*}=A_{\bar{x}} \text { and } A_{x}^{2}=(x, \bar{x}) I ;
$$

and consequently, $\left(A_{x}, A_{y}\right)=(x, y)$ and $\|x\| \leqslant\left\|A_{x}\right\| \leqslant \sqrt{2}\|x\|$. Moreover, identifying $H$ with its image $\mathcal{U}$ under the map $x \rightarrow A_{x}$, we have 


$$
\mathscr{I}_{0}=\left\{x \in H:\|x\|^{2}+\sqrt{\|x\|^{4}-\|(x, \bar{x})\|^{2}}<1\right\} .
$$

Thus each of the Cartan domains of type IV is the open unit ball of a finite dimensional Cartan factor of type IV. (See [19, p.48].) Verification of the unproved assertions above is given at the end of this section.

If $\mathscr{U}$ and $\mathscr{B}$ are $\mathrm{J}^{*}$-algebras, the product space $\mathscr{U} \times \mathscr{Z}$ can be made into a $\mathrm{J}^{*}$-algebra in a natural way. Indeed, if $\mathcal{U}$ and $\mathcal{Z}$ are subspaces of $\mathcal{L}(H, K)$ and $\mathcal{L}\left(\mathrm{H}^{\prime}, \mathrm{K}^{\prime}\right)$, respectively, define

$$
\mathscr{I} \times \mathbb{B}=\{(A, B): A \in \mathscr{Y}, B \in \mathcal{B}\},
$$

where $(A, B)$ is the operator from the Hilbert space $H \times H^{\prime}$ to the Hilbert space $K \times K^{\prime}$ given by $(A, B)(x, y)=$ $(A x, B y)$. Clearly $\mathscr{Z} \times \mathbb{Z}$ is a $\mathrm{J}^{*}$-algebra and it is easy to show that

$$
\|(A, B)\|=\max \{\|A\|,\|B\|\} .
$$

There is no difficulty in extending this definition and equality (4) to the case of finite or infinite products. Thus in particular, any product of open unit balls of $\mathrm{J}^{*}$-algebras is the open unit ball of the corresponding product of the $\mathrm{J}^{*}$-algebras, which is itself a $\mathrm{J}^{*}$-algebra.

Clearly $\mathrm{J}^{*}$-algebras are not algebras in the ordinary sense; however, as the following proposition shows, $\mathrm{J}^{*}$-algebras do contain certain symmetrically formed products of their elements.

Proposition 1. Let $\mathrm{A}, \mathrm{B}, \mathrm{C} \in \mathcal{\mathscr { I }}$ and let $\mathrm{p}$ be any polynomial. Then
(a) $\mathrm{AB} * \mathrm{C}+\mathrm{CB}^{*} \mathrm{~A} \in \mathcal{U}$,
(b) $A(B * A)^{n}=(A B *)^{n} A \in \mathscr{U}$,
(c) $\mathrm{p}\left(\mathrm{AB}^{*}\right) \mathrm{C}+\mathrm{Cp}\left(\mathrm{B}^{*} \mathrm{~A}\right) \in \mathfrak{U}$,
(d) $\mathrm{p}\left(\mathrm{AB}^{*}\right) \mathrm{Cp}\left(\mathrm{B}^{*} \mathrm{~A}\right) \in \mathcal{U}$.

Proof. Part (a) follows from the identities

$$
\begin{aligned}
& 4 A B^{*} A=\sum_{k=0}^{3}(-1)^{k}\left(B+i^{k} A\right)\left(B+i^{k} A\right)^{*}\left(B+i^{k} A\right) \\
& A B^{*} C+C B^{*} A=(A+C) B^{*}(A+C)-A B^{*} A-C B^{*} C,
\end{aligned}
$$

and part (b) follows from the identity

$$
A(B * A)^{n}=A\left[B(A * B)^{n-1}\right]^{*} A,
$$

part (a) and induction. To prove part (c), it suffices to observe that the operator

$$
\left(A B^{*}\right)^{n} C+C\left(B^{*} A\right)^{n}=A\left[\left(B A^{*}\right)^{n-1} B\right]{ }^{*} C+C\left[B\left(A^{*} B\right)^{n-1}\right]^{*} A
$$

is an element of $\mathcal{U}$ by parts (a) and (b). Finally, part (d) follows from part (c) and the identity 


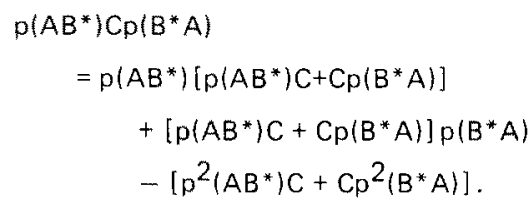

A map $L: \mathscr{I} \rightarrow \mathscr{B}$ is said to be $\mathrm{J}^{*}$-isomorphism if $\mathrm{L}$ is a bounded linear bijection of $\mathscr{U}$ onto $B$ satisfying

$$
L\left(A A^{*} A\right)=L(A) L(A) * L(A)
$$

for all $A \in \mathscr{U}$. Note that the identities given in the proof of Proposition 1 show that any linear map $L: \mathscr{U} \rightarrow B$ satisfying (5) commutes with each of the formulas $(a)-(d)$.

Every $\mathrm{J}^{*}$-algebra is isometrically $\mathrm{J}^{*}$-isomorphic to a $\mathrm{J}^{*}$-algebra of operators on a Hilbert space. In fact, if $H$ and $K$ are Hilbert spaces, the map $L: \mathcal{L}(H, K) \rightarrow \mathcal{L}(H \times K)$ defined by $L(A)(x, y)=(0, A x)$ is a linear isometry satisfying (5).

Certain kinds of operators in $\mathscr{U}$ and relations between them can be characterized entirely in terms of the $\mathrm{J}^{*}$-structure and thus are preserved under $\mathrm{J}^{*}$-isomorphisms. For example, an operator $\mathrm{B}$ is a partial isometry $[25, p .111]$ if and only if $B B^{*} B=B$. Another example, which will be useful later, is the equivalence of the conditions

$$
A^{*} B=0 \text { and } B A^{*}=0 \text {, }
$$

$$
B A^{*} A+A A^{*} B=0
$$

To prove this, all we must show is that $\left(6^{\prime}\right) \Rightarrow(6)$. Suppose $\left(6^{\prime}\right)$ holds. Put

$$
P=\left(B A^{*}\right)\left(B A^{*}\right)^{*}, Q=A A^{*}, R=B B^{*}
$$

and note that each of these operators is positive. Multiplying equation $\left(6^{\prime}\right)$ on the right by $B^{*}$, we have $P+Q R=0$; and taking adjoints we obtain $Q R=R Q$, so both $P$ and $Q R$ are positive. Therefore $P=0$, and consequently, $\mathrm{BA}^{*}=0$. Similarly, multiplying equation $\left(6^{\prime}\right)$ on the left by $\mathrm{B}^{*}$, we obtain $A^{*} B=0$. This completes the proof. Another example of interest is the equivalence of the conditions

$$
C B^{*} A=A B^{*} C=C \text { for all } C \in \mathscr{Y} \text {, }
$$

$$
A B^{*} C+C B^{*} A=2 C \text { and } A B^{*} C B^{*} A=C \text { for all } C \in \mathscr{\Upsilon}
$$

which is easily proved. If the underlying Hilbert spacestand $K$ for $\mathscr{U}$ are chosen so that they are no larger 
than necessary, condition (7) asserts that $B^{*}$ is the inverse of $A$.

Note that the operator norm satisfies

$$
\left\|A A^{*} A\right\|=\|A\|^{3}
$$

for all $A \in \mathcal{L}\left(H_{2} K\right)$, since

$$
\left\|A A^{*} A\right\|^{2}=\left\|\left(A A^{*} A\right)^{*}\left(A A^{*} A\right)\right\|=\left\|(A * A)^{3}\right\|=\left\|A^{*} A\right\|^{3}=\|A\|^{6}
$$

Hence any bounded linear map L: $\mathscr{U} \rightarrow \&$ which satisfies $(8)$ also satisfies $\|L\| \leqslant 1$, since

$$
\|L(A)\|^{3}=\left\|L(A) L(A)^{*} L(A)\right\|=\left\|L\left(A A^{*} A\right)\right\| \leqslant\|L\|\left\|A A^{*} A\right\|=\|L\|\|A\|^{3}
$$

for all $A \in \mathcal{Y}$. Consequently, by the closed graph theorem, any $J^{*}$-isomorphism is an isometry $1 \mathrm{cf}$. [20, p.330]). A converse to this is given in Theorem 4 below.

The rest of this section will be devoted to a further discussion of the Cartan factors of type IV, which are of special interest.

A Cartan factor of type IV can be defined, alternately, as the closed linear space spanned by a spin system [38], i.e., a family $\left\{U_{\alpha}\right\}$ of self-adjoint unitary operators on a Hilbert space such that $\mathrm{U}_{\alpha} \mathrm{U}_{\beta}+\mathrm{U}_{\beta} \mathrm{U}_{\alpha}=0$ when $\alpha \neq \beta$. For, it is easy to see that any such space is a Cartan factor of type IV. Conversely, as we have seen, any Cartan factor of type IV is a Hibert space with conjugation and hence has an orthonormal basis $\left\{U_{\alpha}\right\}$ of self-conjugate elements. Thus $\left\{U_{\alpha}\right\}$ is a spin system and its closed span is the Cartan factor. Spin systems with any given finite number of elements can be constructed explicitly by taking certain Kronecker products of the Pauli spin matrices. Indeed, the set of all $2^{n} \times 2^{n}$ matrices

$$
\underbrace{P x \ldots \times P}_{k} \times Q \times \underbrace{|x \cdots \times|}_{n-k-1}, k=0, \ldots n
$$

where $P=\left(\begin{array}{cc}1 & 0 \\ 0 & -1\end{array}\right)$ and $Q=\left(\begin{array}{ll}0 & 1 \\ 1 & 0\end{array}\right)$ or $\left(\begin{array}{ll}0 & i \\ -i & 0\end{array}\right)$, is a spin system having $2 n+1$ elements.

Let $H$ be a Hilbert space with conjugation $x \rightarrow \bar{x}$, and let $x \rightarrow A_{x}$ be a linear map on $H$ satisfying (2). Given $x, y \in H$, let $\lambda_{1}$ and $\lambda_{2}$ be the roots of the polynomial $p(\lambda)=\lambda^{2}-2(x, \bar{y}) \lambda+(x, \bar{x})(y, \bar{y})$. It will be useful to know that

$$
\sigma\left(A_{y} A_{x}\right)=\left\{\lambda_{1}, \lambda_{2}\right\}
$$

where $\sigma$ denotes the spectrum. To see this, put $A=A_{y} A_{x}$ and $B=A_{x} A_{y}$, and note that $A B=B A$ and $(\lambda I-A)(\lambda I-B)=p(\lambda) I$. Hence $\sigma(A) \subseteq\left\{\lambda_{1}, \lambda_{2}\right\}$. Also if $\lambda_{1} \notin \sigma(A)$, it follows that $B=\lambda_{1} I$, so 
$A=2(x, \bar{y}) 1-B=\lambda_{2} I$. But then $\lambda_{1} \neq \lambda_{2}$ and $\lambda_{2} A_{x}=A_{x} A=B A_{x}=\lambda_{1} A_{x}$, a contradiction. Thus (9) holds. In particular, since $\left\|A_{x}\right\|^{2}$ is the largest number in $\sigma\left(A_{x}^{*} A_{x}\right)$, we have

$$
\left\|A_{x}\right\|^{2}=\|x\|^{2}+\sqrt{\|x\|^{4}-\|(x, \bar{x})\|^{2}}
$$

which justifies (3). Also, it follows from the above proof that

$$
\left(I+A_{y}^{*} A_{x}\right)^{-1}=\frac{I+A_{x} A_{y}^{*}}{1+2(x, y)+(x, \bar{x})(\bar{y}, y)}
$$

Next, following Chevalley $[5, p .38]$, we construct a linear map $x \rightarrow A_{x}$ on $H$ satisfying (2). (Such a map is a complex analogue of what Segal [34] has called a Clifford distribution over $\mathrm{H}$, which extends a construction of Cartan $[4, \S 93]$.) Let $E$ be the exterior algebra of $H$ and note that $E$ is an inner product space [13, p.106]. Given $x \in H$, let $\delta_{x}$ be the antiderivation $[13, p .112]$ of E satisfying $\delta_{x} y=(x, \bar{y}) 1$ for $v \in H$ and define $\ell_{x}(e)=x \wedge$ e for all $e \in E$. Then $\ell_{x}^{2}=0, l_{x} \delta_{x}+\delta_{x} \ell_{x}=(x, \bar{x}) I$, and $\ell_{x}^{*}=\delta_{\bar{x}}$. Define $A_{x}=l_{x}+\delta_{x}$. Thus $A_{x}$ is a linear map on $E$ and the map $x \rightarrow A_{x}$ is linear. Moreover, the above equalities imply that (2) holds, and $A_{X}$ is bounded on $E$ since

$$
\left\|A_{x} e\right\|^{2} \leqslant\left(\left\langle A_{x}^{*} A_{x}+A_{x} A_{x}^{*}\right) e, e\right)=2\|x\|^{2}\|e\|^{2}
$$

for all $e \in E$. Consequently, each operator $A_{X}$ extends uniquely to the completion $K$ of $E$, and thus the map $x \rightarrow A_{x}$ is as required.

Alternately, suppose we already have a spin system $\left\{U_{\alpha}\right\}$ with the same cardinality as the dimension of $H$. Then there is a bijection between an orthonormal basis for $H$ of self-conjugate elements and $\left\{U_{\alpha}\right\}$, and it follows from the last inequality that this bijection extends to a linear map $x \rightarrow A_{x}$ on $H$ with the required properties.

\section{§3. Möbius transformations and biholomorphic mappings}

Theorem 2. For each $B \in \mathfrak{U}_{0}$, the Möbius transformation

$$
\mathrm{T}_{\mathrm{B}}(\mathrm{A})=(\mathrm{I}-\mathrm{BB})^{-1 / 2}(\mathrm{~A}+\mathrm{B})(\mathrm{I}+\mathrm{B} * \mathrm{~A})^{-1}(\mathrm{I}-\mathrm{B} * \mathrm{~B})^{1 / 2}
$$

is a biholomorphic mapping of $\mathscr{U}_{0}$ onto itself with $\mathrm{T}_{\mathrm{B}}(0)=\mathrm{B}$. Moreover,

$$
T_{B}^{-1}=T_{-B}, T_{B}(A)^{*}=T_{B^{*}}\left(A^{*}\right),\left\|T_{B}(A)\right\| \leqslant T_{\|B\|}(\|A\|),
$$

and

$$
\mathrm{DT}_{\mathrm{B}}(\mathrm{A}) \mathrm{C}=(\mathrm{I}-\mathrm{BB})^{1 / 2}(\mathrm{I}+\mathrm{AB})^{-1} \mathrm{C}\left(\mathrm{I}+\mathrm{B}^{*} \mathrm{~A}\right)^{-1}(\mathrm{I}-\mathrm{B} * \mathrm{~B})^{1 / 2}
$$

for $\mathrm{A} \in \mathfrak{U}_{0}$ and $\mathrm{C} \in \mathscr{U}$. 
Here positive and negative square roots are defined by the usual power series expansions and $I$ at each occurrence denotes the identity mapping on the appropriate underlying Hilbert space.

Corollary 2. The open unit ball of any $\mathrm{J}^{*}$-algebra is a bounded symmetric homogeneous domain.

Example 1. Let $C(S)$ be the space of all continuous complex-valued functions vanishing at infinity on a locally compact Hausdorff space $S$. Then with the identification mentioned in $\$ 2$, the space $C(S)$ is a $J^{*}$ algebra and we have

$$
T_{y}(x)=\frac{x+y}{1+\bar{y} x}
$$

for $x, y \in C(S)_{0}$. Note that if $S$ is a discrete set with exactly $n$ elements, then $C(S)_{0}$ is the open unit polydisc in $\mathrm{C}^{n}$. (One may also view the open unit polydisc in $\mathrm{C}^{n}$ as the open unit ball of the $\mathrm{J}^{*}$-algebra of all $\mathrm{n \times n}$ diagonal matrices with complex entries.)

Example 2. Let $H$ be a Hilbert space and let $y \in H_{0}$. Then identifying $H$ with $\mathcal{L}(C, H)$, we have $y^{*} w=(w, y)$ for $w \in H$. Let $E_{y}$ be the linear projection of $H$ onto the subspace spanned by $y$. Then by the power series expansion,

$$
\left(I-y{ }^{*}\right)^{-1 / 2}=\left(I-\|y\|^{2} E_{y}\right)^{-1 / 2}=I+\left[\left(1-\|y\|^{2}\right)^{-1 / 2}-1\right] E_{y}
$$

so

$$
T_{y}(x)=\frac{y+E_{y} x+\sqrt{1-\|y\|^{2}}\left(1-E_{y}\right) x}{1+(x, y)}
$$

for $x \in H_{0}$. When $H$ is finite dimensional, $H_{0}$ is sometimes referred to as a hyperball.

Example 3. Let $H$ be a Hilbert space with conjugation $x \rightarrow \bar{x}$. Then $H$ can be identified (after renorming) with a Cartan factor $\mathscr{X}$ of type IV and $\mathscr{U}_{0}$ is given by (3). Let $y \in \mathscr{U}_{0}$ and let $E_{y}$ be the projection of $H$ onto the space spanned by $y$ and $\bar{y}$. (Explicitly,

$$
E_{y}=\frac{\left(y y^{*}-\bar{y} \bar{y}^{*}\right)^{2}}{\|y\|^{4}-\|(y, \bar{y})\|^{2}}
$$

if $y$ and $\bar{y}$ are linearly independent.) Then by (1), (10) and a computation, we have

$$
T_{y}(x)=\frac{[1+(x, y)] y+[(x, \bar{x})+(x, \bar{y})] \bar{y}+L_{y} x}{1+2(x, y)+(x, \bar{x})(\bar{y}, y)}
$$

for $x \in \mathscr{U}_{0}$, where

$$
L_{y}=\left(1-\|y\|^{2}\right) E_{y}+\sqrt{\left.1-2\|y\|^{2}+\| y, \bar{y}\right) \|^{2}}\left(1-E_{y}\right)
$$

When $H$ is finite dimensional, $\mathscr{U}_{0}$ is sometimes referred to as a Lie ball. 
As in the case of one complex variable, one can use the Möbius transformations together with the Schwarz lemma and Cauchy estimates to prove function-theoretic inequalities. In particular, Theorems 7 and 11 and Corollaries 5 and 6 of [18] hold for all $\mathrm{J}^{*}$-algebras. Further, with the aid of Theorems 1 and 2 , one can show that

$$
d(A, B)=\tanh ^{-1}\left(I I T_{-B}(A) \|\right)
$$

is a complete metric on $\mathscr{U}_{0}$ and that the Schwarz-Pick inequality holds for $d$. See [9] for a generalization.

The formula for the Möbius transformations of Theorem 2 is apparently due to Potapov [29; Ch.1, \$1], although he considered these transformations in a much more restrictive setting. The formulas deduced in Examples 2 and 3 appear in a slightly different form in [27] and [24, (16)], respectively. For expositions of the classical theory of biholomorphic mappings and homogeneous domains in $\mathrm{C}^{n}$, see [1], [10], and [26] .

Proof of Theorem 2. Clearly $\left(1-B^{*}\right)^{1 / 2} B=B\left(I-B^{*} B\right)^{1 / 2}$ by comparison of the power series expansions. Hence since

$$
(A+B)\left(I+B^{*} A\right)^{-1}=B+\left(I-B B^{*}\right) A\left(I+B^{*} A\right)^{-1}
$$

we have

$$
T_{B}(A)=B+\left(I-B B^{*}\right)^{1 / 2} A\left(I+B^{*} A\right)^{-1}\left(I-B^{*} B\right)^{1 / 2}
$$

for $A \in \mathfrak{Y}{ }_{0}$. Now the power series expansions for $A\left(I+B^{*} A\right)^{-1},\left(I-B B^{*}\right)^{1 / 2}$ and $\left(I-B^{*} B\right)^{1 / 2}$ converge in the operator norm. Hence $A\left(I+B^{*} A\right)^{-1} \in \mathcal{X}$ by part (b) of Proposition 1, and consequently $T_{B}\left(\mathscr{U}_{0}\right) \subseteq \mathcal{U}^{\prime}$ by part (d) of the same proposition. Differentiation of (11) shows that $T_{B}$ has the derivative asserted, so $T_{B}$ is holomorphic in $\mathscr{U}_{0}$. Also it is clear from (11) that $T_{B}(0)=B$ and that $T_{B}(A)^{*}=T_{B^{*}}\left(A^{*}\right)$.

We will deduce the inequality $\left\|T_{B}(A)\right\| \leqslant T_{\|B\|}$ (IAII) from the formula

$$
I-T_{B}(A)^{*} T_{B}(A)=\left(I-B^{*} B\right)^{1 / 2}(I+A * B)^{-1}\left(I-A^{*} A\right)\left(I+B^{*} A\right)^{-1}\left(I-B^{*} B\right)^{1 / 2}
$$

which follows as in $[29 ;$ Ch.1, §1]. Let $H$ be as in Definition 1 and let $x \in H$. Taking $y=\left(I+B^{*} A\right)^{-1}\left(I-B^{*} B\right)^{1 / 2} x$ and applying $(12)$, we have that

$$
\begin{gathered}
\left(\left[I-T_{B}(A) * T_{B}(A)\right] x, x\right)=\left(\left(1-A^{*} A\right) y, y\right) \geqslant\left(1-\|A\|^{2}\right)\|y\|^{2} \\
\geqslant\left(1-\|A\|^{2}\right)(1+\|B\|\|A\|)^{-2}\left(1-\|B\|^{2}\|x\|^{2}\right. \\
=\left[1-T_{\|B\|}(\|A\|)^{2}\right]\|x\|^{2}
\end{gathered}
$$

The desired inequality follows.

Thus $T_{B}\left(\mathscr{U}_{0}\right) \subseteq \mathscr{U}_{0}$ and $T_{B}$ is holomorphic in $\mathscr{U}_{0}$. Therefore to finish the proof of Proposition 1, it suffices to show that the function $h=T_{B} \circ T_{-B}$ is the identity map I on $\mathscr{2}_{0}$. By what we have already 
shown, $h$ is a holomorphic mapping of $\mathscr{U}_{0}$ into itself and $D h(0)=D T_{B}(-B) \circ D T_{-B}(0)=I$. Hence an extension of Cartan's uniqueness theorem [14, Th.1] applies to show that $h=I$, as required.

Theorems 1 and 2 allow us to give a characterization of all biholomorphic mappings between the open unit bails of $\mathrm{J}^{*}$-algebras.

Theorem 3. Every biholomorphic mapping $h: \mathscr{I}_{0} \rightarrow \mathscr{S}_{0}$ is of the form

$$
h=T_{h(0)^{\circ L}=L^{\circ} T_{-h^{-1}}(0)},
$$

where $\mathrm{L}: \mathcal{Y} \rightarrow \frac{2}{\mathrm{Y}}$ is a surjective linear isometry.

Proof. By Theorem 2, the composition $\mathrm{T}_{\mathrm{h}(0)}^{-1}$ oh is a biholomorphic mapping of $\mathfrak{2}_{0}$ onto $\mathfrak{B}$ which takes zero to zero. Hence by Theorem 1, there is a surjective linear isometry $L: \mathcal{U} \rightarrow 2$ with $T_{h(0)}^{-1}$ oh $=L$, i.e., $h=T_{h}(0)^{\circ L}$. Since

$$
L\left(h^{-1}(0)\right)=T_{h(0)}^{-1}(0)=-h(0)
$$

by (14) below

$$
\operatorname{LoT}_{-h^{-1}(0)}=T_{h(0)} \circ=h
$$

as asserted.

In particular, Theorem 3 implies that any biholomorphic mapping $\mathrm{h}: \mathscr{I}_{0} \rightarrow \mathfrak{B}_{0}$ is uniquely determined by the first two terms in its Taylor series expansion about 0 . To see this, observe that

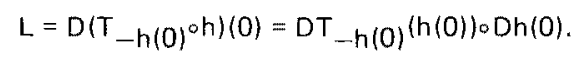

By Theorem 3, to obtain an explicit formula for all biholomorphic mappings between the open unit balls of two $\mathrm{J}^{*}$-algebras, it suffices to obtain an explicit formula for the surjective linear isometries between the $\mathrm{J}^{*}$-algebras. This has been done in certain cases. (See, for example, [8, p.442] , [12, §3], [24], and [28].) In general, surjective linear isometries between $J^{*}$-algebras can be complicated. Even in the case of the finite dimensional Cartan factors, there are surjective linear isometries which are not included in expressions given by Hua $[19, \$ 4.3]$ and Siegel $[36, \$ 48]$ for the biholomorphic mappings of the open unit ball. (For example, the map $A \rightarrow A^{t}$ is a surjective isometry of the Cartan factors of type I with $H=K$. $A$ more subtle example is given by Morita [23] .) However, we do have

Theorem 4. If $L: \mathfrak{I} \rightarrow \mathfrak{B}$ is a surjective linear isometry, then 
for all $A, B \in \mathscr{U}$.

Corollary 3. The domains $\mathscr{U}_{0}$ and $\mathscr{Z}_{0}$ are holomorphically equivalent if and only if the $J *_{- \text {algebras }} \mathfrak{U}$ and $\$$ are isometrically $J^{*}$-isomorphic.

Corollary 4. (Kadison [20]). Let $\mathscr{Y}$ and be $C^{*}-$ algebras each having an identity, and let L: $\mathscr{Y} \rightarrow \mathfrak{B}$ be a surjective linear isometry. Then

$$
\mathrm{L}(\mathrm{A})=\mathrm{U} \rho(\mathrm{A})
$$

for all $A \in \mathscr{U}$, where $U$ is a unitary operator in $\mathcal{Z}$ and $\rho: \mathscr{U} \rightarrow \mathfrak{Z}$ is a linear isometry satisfying

$$
\rho(\mathrm{I})=\mathrm{I}, \quad \rho\left(\mathrm{A}^{2}\right)=\rho(\mathrm{A})^{2}, \text { and } \rho\left(\mathbf{A}^{*}\right)=\rho(\mathrm{A})^{*}
$$

for all $A \in \mathscr{Q}$.

Corollary 5. Let $H$ be a Hilbert space with conjugation $x \rightarrow \bar{x}$ and let $L$ be an invertible linear operator on $H$ which maps the domain ( 3 ) on to itself. Then $L=\lambda O$, where $|\lambda|=1$ and $O$ is a unitary operator on $H$ satisfying $O \bar{x}=\overline{O x}$ for all $x \in H$.

Note that Theorem 4, the identity (11) and our remarks after Proposition 1 combine to show that any surjective linear isometry $L: \mathscr{U} \rightarrow \mathcal{B}$ satisfies

$$
L \circ T_{B}=T_{L(B)} \circ L
$$

for $B \in \mathbb{U}_{0}$

Proof of Theorem 4. Let $B \in \mathscr{Y}_{0}$ and set

$$
h=T_{-L(B)^{\circ}} \operatorname{LoT}_{B}
$$

By hypothesis and the properties of the Möbius transformations, we have that h: $\mathscr{I}_{0} \rightarrow \mathbb{B}_{0}$ is a biholomorphic mapping with $h(0)=0$. Therefore $h$ is linear by Theorem 1, so Dh $(0)=\mathrm{Dh}(-\mathrm{B})$. Applying the chain rule and the formula for the derivative of the Möbius transformations given in Theorem 2, we have

$$
\begin{aligned}
& {\left[I-L(B) L(B)^{*}\right]^{-1 / 2} L\left(\left(I-B B^{*}\right)^{1 / 2} A\left(I-B^{*} B\right)^{1 / 2}\right)\left[I-L(B)^{*} L(B)\right]^{-1 / 2}} \\
& \quad=\left[I-L(B) L(B)^{*}\right]^{1 / 2} L\left(\left(I-B B^{*}\right)^{-1 / 2} A\left(I-B^{*} B\right)^{-1 / 2}\right)\left[I-L(B)^{*} L(B)\right]^{1 / 2}
\end{aligned}
$$


for any $A \in \mathcal{Y}$. Hence by part (d) of Proposition 1,

$$
L\left(\left(I-B B^{*}\right) A\left(I-B^{*} B\right)\right)=\left[I-L(B) L(B)^{*}\right] L(A)\left[I-L(B)^{*} L(B)\right]
$$

for any $A \in \mathscr{U}$. Replacing $B$ in the last equation by $t B$ (where $0<t<1$ ) and then equating the coefficients of $t^{2}$ on both sides, we have

$$
L\left(B B^{*} A+A B * B\right)=L(B) L(B)^{*} L(A)+L(A) L(B) * L(B),
$$

and clearly this holds for all $A, B \in \mathscr{U}$. Theorem 4 now follows from the above with $A=B$ and the first identity given in the proof of Proposition 1.

Proof of Corollaries 3-5. Corollary 3 is an immediate consequence of Theorem 4 and Corollaries 1 and 2. To prove Corollary 4 , let $U=L(I)$ and define $\rho(A)=U^{*} L(A)$. By Theorem 4 and the equivalence of $(7)$ and $\left(7^{\prime}\right)$, we have that $U$ is a unitary operator in $B$. Hence $L(A)=U \rho(A)$ and $\rho(I)=I$. Applying Theorem 4 to the products $A I^{*} A$ and $I A^{*} I$, we obtain $\rho\left(A^{2}\right)=\rho(A)^{2}$ and $\rho\left(A^{*}\right)=\rho(A)^{*}$, respectively.

Finally, to prove Corollary 5 , note that as shown in $\$ 2$, there is a linear bijection $x \rightarrow A_{x}$ satisfying (2) which maps $H$ onto a Cartan factor $\mathscr{U}$ of type IV. Hence $L$ induces a linear map $\tilde{L}: \mathcal{U} \rightarrow \mathscr{U}$, and by hypothesis $\widetilde{L}$ is a surjective isometry. Let $u \in H$ with llull $=1$ and $\bar{u}=u$. Then $A_{u}$ is a unitary operator in $\mathcal{U}$, so as above, $\widetilde{L}\left(A_{U}\right)$ is also a unitary operator in $\widetilde{\mathscr{U}}$. It follows that $\tilde{L}\left(A_{U}\right)=\lambda A_{v}$, where $|\lambda|=1$ and $v \in H$ with $\|v\|=1$ and $\bar{v}=v$. Hence $L u=\lambda v$. By dividing $L$ by $\lambda$, we may suppose that $L u=v$.

Now since $A_{x} A_{y}^{*} A_{x}=2(x, y) A_{x}-(x, \bar{x}) A_{y}^{*}$ by $(1)$, Theorem 4 applied to $\widetilde{L}$ shows that

$$
2[(x, y)-(L x, L y)] L x=(x, \bar{x}) L \bar{y}-(L x, \overline{L x}) \overline{L y}
$$

for all $x, y \in H$. Taking $y=u$ in (15) and using the fact that $L$ is one-to-one, we have

$$
2[(x, u)-(L x, L u)] x=[(x, \bar{x})-(L x, \overline{L x})] u,
$$

and consequently $(L x, \overline{L x})=(x, \bar{x})$ for all $x \in H$. Given $y \in H$, this implies that $(L y, L u)=(y, u)$, and hence taking $x=u$ in (15), we obtain

$$
L \bar{y}-\overline{L y}=2[(u, y)-(L u, L y)] L u=0 .
$$

Thus $\mathrm{L}=\mathrm{O}$.

To exclude complicated surjective linear isometries, following R. S. Phillips [28], we now restrict our attention to the identity component of the group $\mathrm{G}\left(\mathscr{U}_{0}\right)$ of all biholomorphic mappings of $\mathscr{U}_{0}$ onto itself, where $\mathrm{G}\left(\mathfrak{Y}_{0}\right)$ has the topology induced by the metric

$$
\mathrm{d}\left(\mathrm{h}_{1}, \mathrm{~h}_{2}\right)=\sup \left\{\left\|\mathrm{h}_{1}(\mathrm{~A})-\mathrm{h}_{2}(\mathrm{~A})\right\|: \mathrm{A} \in \mathbb{\mathbb { N }}_{0}\right\}
$$


Theorem 5. Let $\mathscr{U}$ be a $\mathrm{C}^{*}$-algebra with identity. Then each function $\mathrm{h}$ in the identity component of $G\left(\mathscr{I}_{0}\right)$ is of the form

$$
h(A)=T_{h(0)}(U A V)=U T_{-h^{-1}(0)}^{(A) V}
$$

where $U$ and $V$ are unitary operators in the weak operator closure of $\mathscr{Y}$.

Corollary 6. Let $\mathfrak{U}$ be a $W^{*}$-algebra. Then the identity component of $G\left(\mathscr{U}_{0}\right)$ is the set of all functions of the form (16) where $U$ and $V$ are unitary operators in $\mathscr{U}$, or equivalently, the set of all functions of the form

$$
Z \rightarrow(A Z+B)(C Z+D)^{-1}
$$

where A, B, C, D are operators in $\mathscr{Y}$ satisfying

$$
\left(\begin{array}{cc}
A & B \\
C & D
\end{array}\right)^{*}\left(\begin{array}{rr}
I & 0 \\
0 & -I
\end{array}\right)\left(\begin{array}{ll}
A & B \\
C & D
\end{array}\right)=\left(\begin{array}{rr}
I & 0 \\
0 & -I
\end{array}\right)=\left(\begin{array}{ll}
A & B \\
C & D
\end{array}\right)\left(\begin{array}{rr}
I & 0 \\
0 & -I
\end{array}\right)\left(\begin{array}{ll}
A & B \\
C & D
\end{array}\right)^{*}
$$

Note that Corollary 6 contains the general symplectic case of Theorem 1 of [28].

Proof of Theorem 5. Let $\mathrm{G}_{\mathrm{e}}$ be the identity component of $\mathrm{G}\left(\mathscr{U}_{0}\right)$, and let $\mathscr{L}_{\mathrm{e}}$ be the identity component of the group $\mathcal{L}$ of all surjective linear isometries $\rho: \mathscr{U} \rightarrow \mathscr{U}$ satisfying (13). We first show that if $h \in \mathrm{G}_{\mathrm{e}}$ then

$$
h(A)=T_{h(0)}(U \rho(A)), A \in \mathscr{U}_{0}
$$

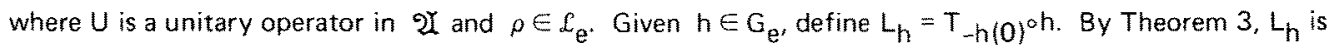
a surjective linear isometry of $\mathscr{U}$ onto itself, and it can be verified that the map $h \rightarrow L_{h}$ is continuous on $G\left(\mathfrak{U}_{0}\right)$. Define $\varphi(h)=L_{h}(I)^{*} L_{h}$. Then by Corollary $4, \varphi$ is a continuous mapping of $G\left(\mathfrak{U}_{0}\right)$ into $\mathcal{L}$ and $\varphi(\mathrm{I})=\mathrm{I}$. Hence $\varphi$ maps $\mathrm{G}_{\mathrm{e}}$ into $\mathcal{L}_{\mathrm{e}^{\prime}}$ and (18) follows.

Now $\mathcal{L}_{e}$ is generated by any of its neighborhoods of I. Hence Theorem 5 follows from (18) and the following:

Lemma 1. If $\rho \in \mathfrak{L}$ and $\|1-\rho\|<2 / 3$, then there is a unitary operator $U$ in the weak operator closure of $\mathscr{U}$ such that $\rho(A)=\mathrm{UAU}^{*}$ for all $\mathrm{A} \in \mathfrak{Y}$.

Proof. By a result of Kadison and Ringrose [21], all we need to show is that $\rho$ is product preserving. By [20], $\rho$ is the sum of $a^{*}$-isomorphism and $a^{*}$-anti-isomorphism, say $\varphi$. In particular, $\varphi$ is a product reversing mapping of $\mathcal{B}$ onto $\mathfrak{F}^{\prime}$, where $\mathcal{H}$ and $\mathcal{Y}^{\prime}$ are $\mathrm{C}^{*}$-subalgebras of $\mathfrak{Y}$, and $\varphi=\rho / \mathcal{Z}$. We will show that 
$\mathcal{S}^{\prime}$ is commutative. It then follows that $\varphi$ is product preserving, and hence so is $\rho$.

Now for any $A, B \in \mathfrak{B}$,

$$
\begin{aligned}
& \|\rho(\mathrm{A}) \rho(\mathrm{B})-\rho(\mathrm{B}) \rho(\mathrm{A})\| \\
& \quad=\|\rho(\mathrm{BA})-\mathrm{BA}+[\mathrm{B}-\rho(\mathrm{B})] \mathrm{A}+\rho(\mathrm{B})\| \mathrm{A}-\rho(\mathrm{A})] \| \\
& \quad \leqslant 3\|\mathrm{I}-\rho\|\|\rho(\mathrm{A})\|\|\rho(\mathrm{B})\|
\end{aligned}
$$

consequently,

$$
\|C D-D C\|<2\|C\|\|D\|
$$

for all nonzero $C, D \in B^{\prime}$. If $\mathfrak{B}^{\prime}$ is not commutative, then as remarked in [6] there is an operator $C \in \mathfrak{Z}$ ' with $C^{2}=0$ and $C \neq 0$. Let $D=C C^{*}-C^{*} C$. Clearly $D \neq 0$ and $\|D\| \leqslant\|C\|^{2}$. Moreover, $\|C D-D C\|=$ $2\left\|\mathrm{CC}^{*} \mathrm{C}\right\|=2\|\mathrm{C}\|^{3}$ by $(8)$. Hence

$$
\|C D-D C\|=2\|C\|\|D\|,
$$

a contradiction. Thus $\mathcal{B}^{\prime}$ is commutative, as we wished to show.

Proof of Corollary 6. An easy argument given in [28, p.17] shows that the set of all transformations (17) is a connected subgroup $S$ of $G\left(\mathscr{U}_{0}\right)$, and it is easy to see that $S$ contains all transformations of the form (16). Hence $S$ is precisely the set of transformations (16) and $S$ is the identity component of $G\left(\mathcal{U}_{0}\right)$.

It seems reasonable to conjecture that Theorem 5 holds for any $\mathrm{J}^{*}$-algebra $\mathscr{\mathcal { X }}$ where $\mathrm{U}$ and $\mathrm{V}$ are unitary operators on the underlying Hilbert spaces. In particular, it follows from Theorem 3, [3, Theorem E] and [24] that this holds for all finite dimensional $J^{*}$-algebras which are products of Cartan factors of types I-IV. (Note that by $[4, \$ 97]$ any rotation of $\mathrm{C}^{n}$ can be represented as a transformation $A \rightarrow U A V$ on the $n-$ dimensional Cartan factor of type IV.)

\section{Factorization of algebras and domains}

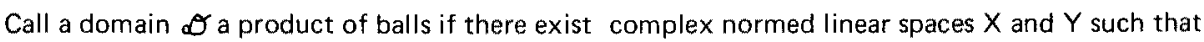
$\mathscr{D}=X_{0} \times Y_{0}$. The following theorem shows that the ball $\mathscr{\varkappa}_{0}$ is holomorphically equivalent to a product of balls if and only if $\mathscr{U}$ is isometrically $\mathrm{J}^{*}$-isomorphic to a product of $\mathrm{J}^{*}$-algebras.

Theorem 6. Let $X$ and $Y$ be complex normed linear spaces and suppose $\mathscr{U}_{0}$ is holomorphically equivalent to $\mathrm{X}_{0} \times \mathrm{Y}_{0}$. Then there exist $\mathrm{J}^{*}$-subalgebras $\mathcal{B}$ and $\mathcal{S}$ of $\mathscr{U}$ which are isometrically isomorphic to $\mathrm{X}$ and $Y$, respectively, such that $\mathscr{Y}$ is isometrically $J^{*}$-isomorphic to the $J^{*}$-algebra $\Re \times \mathfrak{S}$.

Corollary 7. Let $\mathscr{U}$ be a $J^{*}$-algebra containing an isometry. Suppose that if $E$ is a projection of the form $E=B * B$ where $B \in \mathscr{Y}$ and if 
for all $\mathrm{A} \in \mathfrak{U}$, then $\mathrm{E}=0$ or $\mathrm{AE}=\mathrm{A}$ for all $\mathrm{A} \in \mathscr{Y}$. Then $\mathfrak{Y}_{0}$ is not holomorphically equivalent to a product of balls.

It is easy to show that the converse of Corollary 7 holds when $\mathscr{U}$ is such that $A B^{*} C \in \mathscr{I}$ whenever $A, B, C \in \mathscr{Y}$.

Proof of Theorem 6. Let the space $X \times Y$ have the norm $\|(x, y)\|=\max \{\|x\|,\|y\|\}$. Then by hypothesis, $\mathscr{U}_{0}$ is holomorphically equivalent to $(X \times Y)_{0}$ so $\mathscr{U}$ is isometrically isomorphic to $X \times Y$ by Corollaries 1 and 2. It follows that there exist complementary projections $E$ and $F$ on $\mathscr{X}$ such that the spaces $\mathfrak{B}=R$ ge $E$ and $\mathfrak{S}=$ Rge $F$ are isometrically isomorphic to $X$ and $Y$, respectively, and

$$
\|A\|=\max \{\|E(A)\|,\|F(A)\|\}
$$

for all $A \in \mathscr{U}$. Given $|\lambda|=1$, define $L_{\lambda}=E+\lambda F$ and note that $L_{\lambda}$ is a linear isometry of $\mathscr{U}$ onto itseif. Hence by Theorem 4 ,

$$
L_{\lambda}\left(A B^{*} A\right)=L_{\lambda}(A) L_{\lambda}(B)^{*} L_{\lambda}(A)
$$

for all $A, B \in \mathcal{U}$. Varying $\lambda$ in $(21)$, we obtain $E\left(A B^{*} A\right)=E(A) E(B)^{*} E(A)$ for all $A, B \in B$ and similarly $F\left(A B^{*} A\right)=F(A) F(B)^{*} F(A)$ for all $A, B \in \mathbb{S}$. Hence $\&$ and $\subseteq$ are $J^{*}$-subalgebras of $\mathscr{U}$, Defining $L(A)=(E(A), F(A))$, we see by $(4)$ and $(20)$ that $L$ is a linear isometry of $\mathscr{X}$ onto the $J^{*}$-algebra $\mathfrak{B} \times(S$. Therefore, $\mathfrak{U}$ and $B \times \mathbb{E}$ are isometrically $\mathrm{J}^{*}$-isomorphic by Theorem 4 .

Proof of Corollary 7. Suppose $\mathfrak{U}_{0}$ is holomorphically equivalent to a product of balls. Then by Theorem 6 , there exist $\mathrm{J}^{*}$-algebras $B$ and $\mathcal{S}$ such that $\mathscr{U}$ is isometrically $\mathrm{J}^{*}$-isomorphic to $\mathbb{B} \times \mathcal{S}$. Now given any two operators $V$ and $A$ in $B \times \subseteq$, there exist operators $V_{1}, V_{2}, A_{1}$, and $A_{2}$ in $\mathscr{B} \times \mathbb{S}$ such that

$$
\begin{gathered}
V=V_{1}+V_{2}, A=A_{1}+A_{2}, \\
V_{1} V_{2}^{*}=A_{1} V_{2}^{*}=A_{2} V_{1}^{*}=A_{1} A_{2}^{*}=0, V_{2}^{*} V_{1}=V_{2}^{*} A_{1}=V_{1}^{*} A_{2}=A_{2}^{*} A_{1}=0 ;
\end{gathered}
$$

moreover, there are operators $A$ for which neither $A_{1}$ nor $A_{2}$ is 0 . Hence by the equivalence of $(6)$ and $\left(6^{\prime}\right)$, the same is true for any two operators $V$ and $A$ in $\mathfrak{U}$. Let $V$ be an isometry. Then

$$
I=V^{*} V=V_{1}^{*} V_{1}+V_{2}^{*} V_{2}
$$

and multiplying both sides of this equation by $E=V_{1}^{*} V_{1}$, we see that $E$ is a projection. Moreover. 
$A_{1}(I-E)=A_{2} E=0$, so

$$
A_{1}=A E, A_{2}=A(I-E)
$$

Since $A_{2}^{*} A_{1}=0$, we have $A^{*} A E=E A^{*} A E$, and taking adjoints we obtain (19). Hence by hypothesis, one of the operators $(22)$ is always 0 , the desired contradiction.

Clearly one can obtain algebraic identities satisfied by any hermitian projection [2] on a $\mathrm{J}^{*}$-algebra by equating the coefficients of the powers of $\lambda$ in (21) where $|\lambda|^{2}$ is replaced by 1 . These identities imply in particular that the identity map is the only hermitian projection $E$ on a $B^{*}$-algebra with identity 1 such that $E(1)=1$.

The rest of this section is devoted to applications of Theorem 6 and its corollary.

Call a $\mathrm{J}^{*}$-algebra $\mathscr{U}$ a finite dimensional Cartan factor if $\mathscr{U}_{0}$ is holomorphically equivalent to one of the Cartan domains of types I-VI. By Corollary 3, we have already determined up to isometric $\mathrm{J}^{*}-$ isomorphism all finite dimensional Cartan factors except possibly for a 16 dimensional one and a 27 dimensional one. (See §2.) Clearly no finite dimensional Cartan factor (except for the 2-dimensional one of type IV) is isometrically $\mathrm{J}^{*}$-isomorphic to a product of $\mathrm{J}^{*}$-algebras, otherwise the associated Cartan domain would be reducible.

Theorem 7. Every finite dimensional $\mathrm{J}^{*}$-algebra is isometrically $\mathrm{J}^{*}$-isomorphic to a product of finite dimensional Cartan factors. In particular, every $\mathrm{J}^{*}{ }_{-}$algebra of dimension less than 16 is isometrically $\mathrm{J}^{*}$ isomorphic to a product of finite dimensional Cartan factors of types I-IV.

Proof of Theorem 7. Let $\mathfrak{U}$ be an $n$-dimensional $J^{*}$-algebra and identify $\mathscr{U}$ with $\mathrm{C}^{n}$. By Corollary 2 and Cartan's classification [3] of bounded symmetric domains, there exist domains $\sigma_{2}, \ldots \infty_{k}$ in the spaces $x_{2}=c^{n_{2}}, \ldots, x_{k}=c^{n_{k}}$, respectively, such that $\mathscr{U}_{0}=\infty_{2} \times \cdots \times \sigma_{k}$, and each $\mathscr{\sigma}_{j}$ is holomorphically equivalent to a Cartan domain of type I-VI. Since $\mathfrak{H}_{0}$ is a balanced convex open subset of $C^{n}$, each $\mathscr{O}_{j}$ is a balanced convex open subset of $X_{j}$ so $\mathscr{O}_{j}$ is the open unit ball of $X_{j}$ with respect to some norm. Then $\mathcal{U}$ is the space $X_{2} \times \cdots \times X_{k}$ with the max norm on coordinates since the open unit balls of these spaces agree. By successive application of Theorem 6 , it follows that each $X_{j}$ is isometrically isomorphic to a $J^{*}-$ algebra $\mathfrak{U}_{j}$ and consequently each $\mathfrak{U}_{j}$ is a finite dimensional Cartan factor. Hence $\mathfrak{U}_{\text {is isometrically }}$ isomorphic to the $\mathrm{J}^{*}$-algebra $\mathscr{\mathscr { U }}_{2} \times \cdots \times \mathscr{\mathscr { U }}_{\mathrm{k}}$, and this isomorphism is a $\mathrm{J}^{*}$-isomorphism by Theorem 4 .

Theorem 8. The open unit balls of the following $\mathrm{J}^{*}$-algebras are not holomorphically equivalent to a product of balls: all Cartan factors of type I-IV except the 2-dimensional one of type IV; all $\mathrm{W}^{*}$-algebras which are factors in the usual sense; all spaces $C(S)$, where $S$ is compact and connected.

For a description of a large number of $W^{*}$-algebras which are factors, see $[33, \S 4]$. 
Proof of Theorem 8. It is easy to see from Corollary 7 that Theorem 8 holds for $W^{*}$-algebras which are factors and for spaces $C(S)$, where $S$ is compact and connected.

Suppose $\mathfrak{U}$ is a Cartan factor of type I, i.e., $\mathscr{U}=\mathcal{L}(H, K)$. There exist conjugations $\mathrm{Q}_{1}$ on $\mathrm{H}$ and $\mathrm{O}_{2}$ on $K$, and clearly the map $A \rightarrow Q_{1} A^{*} Q_{2}$ is a linear isometry of $\mathcal{L}(H, K)$ onto $\mathcal{L}(K, H)$. Hence by the compara bility of cardinal numbers, it suffices to consider only the case where $\operatorname{dim} H \leqslant \operatorname{dim} K$. Note that in this case $\mathcal{L}(H, K)$ contains an isometry. Suppose $E$ is a projection in $\mathcal{L}(H)$ satisfying (19). Given $x \in H$ and $y \in K$ with $\|y\|=1$, take $A=y x^{*}$. Then $A^{*} A=x x^{*}$, so,

$$
0=E A * A(I-E) x=\|(I-E) x\|^{2} E x .
$$

Consequently, $E x=0$ or $E x=x$ for each $x \in H$, and it is easy to show that this implies that $E=0$ or $E=I$.

Suppose $\mathscr{U}$ is a Cartan factor of type II. Clearly $I \in \mathscr{U}$. Suppose $E$ is a projection in $\mathcal{L}(H)$ satisfying (19), and let

$$
H_{r}=\{x \in H: \bar{x}=x\} \text {, }
$$

where $Q(x)=\bar{x}$ is the given conjugation on $H$. Then given $x \in H_{r}$, the operator $A=x x^{*}$ satisfies $A^{t}=A$ so $A \in \mathscr{U}$. The argument given for Cartan factors of type I now applies to show that $E=0$ on $H_{r}$ or $E=I$ on $H_{r}$. But $H=H_{r}+i H_{r}$, so $E=0$ or $E=I$.

Suppose $\mathscr{U}$ is a Cartan factor of type III. Since Theorem 8 is classical [3] when $\mathrm{H}$ is finite dimensional, we may suppose that $\mathrm{H}$ is infinite dimensional. Then there is an orthonormal basis for $\mathrm{H}_{\mathrm{r}} \mathrm{H}_{\mathrm{r}}$ and this basis can be partitioned into two equivalent sets $\left\{e_{\alpha}\right\}$ and $\left\{e_{\alpha}^{\prime}\right\}$. Let $U$ be the operator on $H$ defined by $\cup e_{\alpha}=e_{\alpha}^{\prime}$ and $U \mathrm{e}_{\alpha}^{\prime}=-\mathrm{e}_{\alpha}$. Then $\mathrm{U}=\mathrm{QUQ}, \mathrm{U}^{2}=-\mathrm{I}$ and $\mathrm{U}^{*}=-\mathrm{U}$. Hence $\mathrm{U}$ is a unitary operator in $\mathscr{Y}$.

Now suppose $E$ is a projection in $\mathcal{L}(H)$ satisfying (19). Let $x$ be a unit vector in $H_{r}$ and extend $x$ to an orthonormal basis for $H_{r}$. This basis is then an orthonormal basis for $H$ and may be partitioned into sets $\{x\}$, $\left\{e_{\alpha}\right\}$, and $\left\{e_{\alpha}^{\prime}\right\}$, where the last two sets are equivalent. Let $A$ be the operator on $H$ defined by $A x=0$, $\mathrm{Ae}_{\alpha}=\mathrm{e}_{\alpha^{\prime}}^{\prime}$ and $\mathrm{Ae}_{\alpha}^{\prime}=-\mathrm{e}_{\alpha}$. Then $\mathrm{A}=\mathrm{QAO}, \mathrm{A}^{*}=-\mathrm{A}$, and $\mathrm{A}^{2}=\mathrm{x} \mathrm{x}^{*}-\mathrm{I}$. Hence $\mathrm{A} \in \mathcal{U}$ and $\mathrm{A}^{*} A=I-x x^{*}$. The argument given for Cartan factors of type II now applies to show that $E=0$ or $E=I$.

Suppose $\mathfrak{U}$ is a Cartan factor of type IV and that $\operatorname{dim} \mathscr{U}>2$. Then $\mathscr{U}$ is the space spanned by a spin system which we write in the form $\left\{U_{0}\right\} \cup\left\{U_{\alpha}\right\}$, where $U_{0}$ is distinct from any of the $U_{\alpha}$ 's. Taking $V_{\alpha}=i U_{0} U_{\alpha}$, we see that $\left\{V_{\alpha}\right\}$ is a spin system with at least two elements and that the $J^{*}$-algebra $\mathfrak{Z}=U_{0} \mathscr{U}$ is the closed space spanned by the set $\{1\} \cup\left\{V_{\alpha}\right\}$. Since $\mathscr{U}$ is isometrically $J^{*}$-isomorphic to $\mathbb{B}$, it suffices to show that $\mathbb{B}$ satisfies the hypotheses of Theorem 6 . Suppose (19) holds. Taking $A=I+V_{\alpha}$ in $(19)$, we have $E V_{\alpha}=V_{\alpha} E$ for all $\alpha$. Then $E B=B E=B$, so

$$
E=\left(B B^{*}+B^{*} B\right)-B^{*} B^{2} B^{*}=2 B
$$

since $\left\{\right.$ contains the squares and adjoints of each of its elements. Hence $V_{\alpha} E+E V_{\alpha}=\lambda_{\alpha} I+\mu_{\alpha} V_{\alpha}$, where $\lambda_{\alpha}$ and $\mu_{\alpha}$ are complex numbers. But since $V_{\alpha} E=E V_{\alpha}$, it follows that $2 E=\mu_{\alpha} I+\lambda_{\alpha} V_{\alpha}$ for all $\alpha$, so $E=0$ or $\mathrm{E}=\mathrm{I}$. 


\section{\$5. Function-theoretic boundaries and extreme points}

In this section we show that the sets defined below play the role of function-theoretic boundaries for the open unit balls of $\mathrm{J}^{*}$-algebras.

Definition 2. A non-empty subset $\Gamma$ of the unit sphere of $\mathfrak{U}$ is said to be stable if the transformation $A \rightarrow T_{B}(\lambda A)$ takes $\Gamma$ to $\Gamma$ for each $B \in \mathscr{U}_{0}$ and each complex number $\lambda$ with $|\lambda|=1$.

Clearly any non-empty intersection of stable sets is stable. Also, any component of a stable set is stable; indeed, suppose $\Gamma^{\prime}$ is a component of a stable subset $\Gamma$ of $\mathscr{Y}$ and let $A \in \Gamma^{\prime}, B \in \mathscr{U}_{0}$ and $|\lambda|=1$. Then the map $t \rightarrow T_{t B}\left(\lambda^{t} A\right)$ on $[0,1]$ is a continuous curve in $\Gamma$ connecting $A$ to $T_{B}(\lambda A)$, so $T_{B}(\lambda A) \in \Gamma^{\prime}$. Therefore, the transformation $A \rightarrow T_{B}(\lambda A)$ takes $\Gamma^{\prime}$ to $\Gamma^{\prime}$, as required. Note that by Theorem 3 and (14), every biholomorphic mapping of $\mathfrak{Y}_{0}$ onto $\mathcal{B}_{0}$ extends to a homeomorphism of $\mathfrak{X}_{1}$ onto $\mathfrak{B}_{1}$ which takes stable sets to stable sets.

Proposition 2. Any of the following subsets of $\mathscr{U}$ is stable if non-empty:

(a) the unitary operators in $\mathscr{U}$, (b) the isometries in $\mathscr{U}$, (c) the extreme points of $\mathfrak{U}_{1}$.

Proof. By definition or by Theorem 11 below, an operator $B \in \mathcal{U}$ is in the sets described in (a), (b), or $(c)$ if and only if both $B^{*} B=I$ and $B B^{*}=1, B^{*} B=I$, or $\left(I-B B^{*}\right) A\left(I-B^{*} B\right)=0$ for all $A \in \mathscr{U}$, respectively. Hence Proposition 2 is an immediate consequence of (12) and the complementary formula

$$
I-T_{B}(A) T_{B}(A)^{*}=\left(I-B B^{*}\right)^{1 / 2}\left(I+A B^{*}\right)^{-1}\left(I-A A^{*}\right)\left(I+B A^{*}\right)^{-1}\left(I-B B^{*}\right)^{1 / 2},
$$

which follows from (12) and the equality

$$
I-T_{B}(A) T_{B}(A)^{*}=I-T_{B}\left(A^{*}\right)^{*} T_{B^{*}}\left(A^{*}\right) \text {. }
$$

There are many $\mathrm{J}^{*}$-algebras for which one of the sets $(\mathrm{a})-(\mathrm{c})$ is non-empty. For example, if $\mathcal{I}$ is a $B^{*}$-algebra with identity, obviously $\mathcal{Y}$ contains a unitary element. Also if $\mathscr{U}=\mathcal{L}(H, K)$, where $H$ and $K$ are (possibly infinite dimensional) Hibert spaces with $\operatorname{dim} \mathrm{H} \leqslant \operatorname{dim} \mathrm{K}$, then $\mathcal{Q}$ contains an isometry. Further, if $\mathscr{U}$ is any finite dimensional $\mathrm{J}^{*}$-algebra or, more generally, any $\mathrm{J}^{*}$-algebra which is closed in the weak operator topology, then $\mathscr{I}_{1}$ has an extreme point. (To see the last assertion, apply the KreinMilman theorem and the fact that $\mathscr{U}_{1}$ is compact in the weak operator topology [8; Ex.6, p.512].)

Let $X$ be a complex normed linear space. The holomorphic hull of a subset $\Lambda$ of $X$ (which we denote by Co $\Lambda$ is defined to be the set of all $x \in X$ such that $\lg (x) \mid \leqslant 1$ whenever $g: X \rightarrow C$ is a holomorphic function satisfying $\lg (y) \mid \leqslant 1$ for all $\mathrm{y} \in \Lambda$. Clearly Co $\Lambda$ is closed, and by a well-known separation theorem [8; Th.10, p.417], it follows that 


$$
\operatorname{Co} \Lambda \subseteq \operatorname{co} \Lambda
$$

where co $\Lambda$ denotes the closed convex hull of $\Lambda$.

We can now state the main result of this section (cf. [17] and [22, Prop. 3.2] .)

Theorem 9 (Maximum principle). Let $\Gamma$ be a stable subset of $\mathscr{U}$ and let $h: \mathscr{U}_{0} \rightarrow X$ be a holomorphic function with a continuous extension to $\mathscr{U}_{0} \cup \Gamma$. Then

$$
h\left(\mathfrak{\varkappa}_{0}\right) \subseteq \operatorname{Coh}(\Gamma)
$$

In particular, if $h$ is bounded on $\Gamma$,

$$
\|h(B)\| \leqslant \sup \{\|h(A)\|: A \in \Gamma\}
$$

for all $B \in \mathscr{\Upsilon}_{0}$. Moreover, $h$ is completely determined by its values on $\Gamma$.

Note that if $S$ is the unit circle, the components of the set of unitary elements of $C(S)$ are just the homotopy classes of continuous mappings of $S$ into itself. Thus $C(S)$ has infinitely many disjoint closed stable subsets.

Letting $h$ be the identity map on $\mathscr{U}$ in $(25)$ and applying $(24)$, we obtain a result which considerably extends the Russo-Dye theorem [32, Th.1].

Corollary 8. If $\Gamma$ is a component of any non-empty one of the sets (a)-(c) of Proposition 2 , then $\mathscr{U}_{1}=c 0 \Gamma$.

For example, let $\mathscr{U}$ be a Cartan factor of type IV and note that all normal operators in $\mathscr{U}$ are scalar multiples of self-adjoint unitary operators in $\mathscr{U}$. By Corollary 8 above and Lemma 2 of [17], the convex hull of the set of all unitary operators in $\mathscr{\mathscr { U }}$ contains $\mathscr{\mathscr { U }}_{0^{\circ}}$ Hence if $\mathrm{H}$ is any Hilbert space with conjugation $x \rightarrow \bar{x}$, the norm whose open unit ball is given by $(3)$ is the largest norm on $H$ which agrees with the Hilbert norm on self-conjugate elements. (This has been observed in the finite dimensional case by Druzkowski [7] .)

In finite dimensional $\mathrm{J}^{*}$-algebras there is a smallest closed stable set. Indeed,

Corollary 9. Suppose $\mathscr{U}$ is finite dimensional. Then the set $\mathscr{E}$ of extreme points of $\mathfrak{U}_{1}$ is the BergmanShilov boundary for $\mathscr{\mathscr { U }}_{0}$, i.e., $\&$ is the smallest closed subset of the unit sphere of $\mathscr{U}$ with the property that every complex-valued function holomorphic in $\mathscr{\mathscr { U }}_{0}$ and continuous in $\mathscr{U}_{1}$ assumes its maximum absolute value on $\mathscr{E}$. Moreover, $\mathscr{E}$ is connected and is contained in the closure of every stable subset of $\mathscr{U}$.

For example, it is known $[19, p .6]$ that the Bergman-Shilov boundary for a classical Cartan domain $\infty$ of 
(a) type I with $\mathrm{n}<\mathrm{m}$ is the set of all isometries in $\mathrm{Cl} . \mathrm{O}$,

(b) type I with $n=m$, type II, type III with n even, or type IV is the set of all unitary operators in $\mathrm{Cl} \infty$

(c) type III with $n$ odd is the set of all operators $V \in C$ C $\mathcal{O}$ satisfying $V^{*} V=I-x x^{*}$ for some $x \in C^{n}$ with $\|x\|=1$.

To see this, apply Theorem 11 below to show that each of the sets described is a set of extreme points of $\mathrm{Cl} \mathscr{O}$ and observe that each of the sets is closed and stable. Note that the Bergman-Shilov boundary for any finite product of the above domains is the corresponding product of the Bergman-Shilov boundaries for each of the domains.

Proof of Theorem 9. Let $g: X \rightarrow C$ be a holomorphic function and suppose $\lg (x) \mid \leqslant 1$ for all $x \in h(\Gamma)$. Given $B \in \mathscr{Y}_{0}$ and $A \in \Gamma$, by hypothesis $T_{B}(\lambda A) \in \Gamma$ whenever $|\lambda|=1$. Hence the function

$$
f(\lambda)=g \circ h\left(T_{B}(\lambda A)\right)
$$

satisfies $|f(\lambda)| \leqslant 1$ for $|\lambda|=1$, and clearly $f$ is holomorphic in the open unit disc and continuous in its closure. Consequently, by the (classical) maximum principle, $\lg (h(B))|=| f(0) \mid \leqslant 1$. Therefore, $h(B) \in$ Co $h(\Gamma)$, which proves (25). Note that (26) follows from (25), (24), and the convexity of balls. The last part of the theorem is immediate from the formula

$$
h(B)=\frac{1}{2 \pi} \int_{0}^{2 \pi} h\left(T_{B}\left(e^{i \theta} A\right)\right) d \theta
$$

which follows from the mean value property for vector-valued holomorphic functions.

Proof of Corollary 9. Let $\Lambda$ be the Bergman-Shilov boundary for $\mathscr{U}_{0}$. (The existence of $\Lambda$ is well known $[10, p .217]$.) By Theorem 9 and the compactness of $\mathscr{U}_{1}$, it follows that $\Lambda$ is contained in the closure of every stable subset of $\mathscr{U}$. In particular, since each component of $\mathscr{E}$ is a stable subset of $\mathscr{U}$ and $\mathscr{E}$ is closed (see Theorem 11 below), we have that $\Lambda \subseteq \&$ and $\&$ is connected. On the other hand, $\mathfrak{Y}_{1} \subseteq \operatorname{Co} \Lambda \subseteq \operatorname{co} \Lambda$ so $\& \subseteq \Lambda$ bya converse to the Krein-Milman Theorem [8; Lemma 5, p.440].

Theorem 10 (Schwarz lemma). Let h: $\mathscr{Y}_{0} \rightarrow X_{1}$ be a holomorphic function with $h(0)=0$ and put $L=D h(0)$. If $L$ takes a stable subset $\Gamma^{*}$ of $\mathscr{L}$ into a set of complex extreme points of $X_{1}$, then $h=L$.

Proof. Given $A \in \Gamma$, define $f(\lambda)=\frac{1}{\lambda} h(\lambda A)$ for $0<|\lambda|<1$ and take $f(0)=L(A)$. Clearly the composition of $f$ with any bounded linear functional on $X$ is holomorphic in the open unit disc $\Delta$, and hence by the (classical) maximum principle and the Hahn-Banach theorem, we have $f(\Delta) \subseteq X_{1}$. Then since $L(A)$ is a complex extreme point of $X_{1}$, it follows that $f$ is constant by the Thorp-Whitiey maximum principle [37] . 
(See [14] for a simple proof which applies to this stightly more general situation.)

Now given $0<r<1$, define $g(B)=h(r B)-L(r B)$ for $B \in \mathscr{U}_{1}$. Then $g$ is continuous in $\mathscr{U}_{1}$ and holomorphic in $\mathscr{U}_{0}$, and by the above $g$ vanishes on $\Gamma$. Hence $g$ vanishes identically in $\mathfrak{U}_{1}$ by Theorem 9. Therefore $h=L$.

Theorem 11. The extreme points of $\mathfrak{U}_{1}$ coincide with the complex extreme points of $\mathfrak{U}_{1}$ and are precisely those operators $B \in \mathscr{U}$ satisfying

$$
\left(I-B^{*}\right) A\left(I-B^{*} B\right)=0
$$

for all $A \in \mathscr{U}$. In particular, every extreme point of $\mathfrak{U}_{1}$ is a partial isometry.

Note that Theorem 11 extends a result of Kadison [20]. For the proof, we first establish an inequality of independent interest.

Proposition 3. If $B \in \mathcal{L}(\mathbf{H}, K)_{1}$, then

$$
\left\|\mathrm{B}+(\mathrm{I}-\mathrm{BB} *)^{1 / 2} \mathrm{~A}(\mathrm{I}-\mathrm{B} * \mathrm{~B})^{1 / 2}\right\| \leqslant 1
$$

for all $\mathbf{A} \in \mathcal{L}(\mathbf{H}, \mathbf{K})$ with $\|\mathrm{A}\| \leqslant 1 / 2$.

Proof. First observe that the functions $B \rightarrow\left(1-B^{*} B\right)^{1 / 2}$ and $B \rightarrow\left(1-B B^{*}\right)^{1 / 2}$ are defined and continuous in $\mathcal{L}(\mathrm{H}, \mathrm{K})$, by the (commutative) Gelfand Naimark theorem [31, Th. 4.2.2] and the uniform convergence of the binomial series for $(1-t)^{1 / 2}$ on $[0,1]$. Hence if $T_{B}(C)$ is defined by $(11)$ for $C \in \mathscr{L}(H, K)_{0}$, Theorem 2 implies that

$$
\left\|T_{B}(C)\right\| \leqslant 1
$$

Given $A \in \mathcal{L}(H, K)$ with $\|A\|<1 / 2$, take $C=A\left(I-B^{*} A\right)^{-1}$. Then $C \in \mathcal{L}(H, K),\|C\| \leqslant\|A\|(1-\|A\|)^{-1}<1$, and $A=C(I+B * C)^{-1}$. Hence Proposition 3 follows from (28).

Proof of Theorem 11. Note that Proposition 3 holds without the exponents, and hence any complex extreme point of $\mathscr{U}_{1}$ satisfies (27). Thus to finish the proof, it suffices to show that any operator $B$ satisfying $(27)$ is a partial isometry and an extreme point of $\mathscr{U}_{1}$. Clearly $B=B B^{*} B$ since

$$
\left(B-B B^{*} B\right)^{*}\left(B-B B^{*} B\right)=B^{*}\left[\left(I-B B^{*}\right) B\left(I-B^{*} B\right)\right]=0 \text {, }
$$

and consequently $E=B^{*} B$ and $F=B B^{*}$ are projections. Hence $B$ is a partial isometry. Suppose $A \in \mathfrak{X}$ and $\|B \pm A\| l \leqslant 1$. Let $x \in H$, where $H$ is as in Definition 1 and set $y=E x$. Then 


$$
\|y\|^{2} \geqslant\|B y \pm A y\|^{2}=\|B y\|^{2} \pm 2 \operatorname{Re}(B y, A y)+\|A y\|^{2} .
$$

Choosing the appropriate sign, we have that $\|y\|^{2} \geqslant\|B y\|^{2}+\|A y\|^{2}$; but $\|B y\|^{2}=\|y\|^{2}$, so $A y=0$. Hence $A E=0$. A similar argument beginning with the inequality $\left\|B^{*} \pm A^{*}\right\| \leqslant 1$ shows that $A^{*} F=0$, i.e., $F A=0$. Hence $A=(I-F) A(I-E)=0$, as required.

\section{§6. A generalization of the upper half-plane}

Note that any bounded linear operator $A$ on a Hilbert space $H$ can be written in the form $A=\operatorname{Re} A+i \operatorname{Im} A$, where

$$
\operatorname{Re} A=\frac{A+A^{*}}{2} \text { and } \operatorname{Im} A=\frac{A-A^{*}}{2 i} \text {, }
$$

and clearly both $\operatorname{Re} A$ and $\operatorname{Im} A$ are self-adjoint operators. Write $A>0$ when $A$ is self-adjoint and there is an $\epsilon>0$ such that $(A x, x) \geqslant \epsilon\|x\|^{2}$ for all $x \in H$.

Theorem 12. Let $\mathscr{U}$ be a $J *-$ algebra containing an isometry $V$. Define

$$
\mathscr{H}=\left\{\mathbf{A} \in \mathscr{U}: \operatorname{Im} \mathbf{V}^{*} \mathbf{A}-\mathbf{A}^{*}\left(\mathbf{I}-\mathbf{V V}^{*}\right) \mathbf{A}>0\right\} .
$$

Then $\mathcal{H}$ is an unbounded convex domain in $\mathscr{U}$ and the Cayley transformation

$$
S(A)=i(A+V)\left(I-V^{*} A\right)^{-1}
$$

is a biholomorphic mapping of $\mathscr{U}_{0}$ onto $\pi$. Moreover, if $\mathrm{V}$ is a unitary operator or if $\mathscr{U}$ is such that $\mathrm{AB}^{*} \mathrm{C} \in \mathcal{U}$ whenever $\mathrm{A}, \mathrm{B}, \mathrm{C} \in \mathscr{\mathcal { U }}$, then $\mathcal{H}$ is affinely homogeneous.

Clearly Cartan factors of type I with $\mathrm{H}=\mathrm{K}$ and all Cartan factors of type II contain the identity operator. Also, Cartan factors of type III with the dimension of $\mathrm{H}$ even or infinite and all Cartan factors of type IV contain a unitary operator. (See the proof of Theorem 8.) Thus the corresponding upper half-planes $\mathcal{H}$ are affinely homogeneous tubular domains. Further, if $\mathrm{H}$ and $\mathrm{K}$ are (possibiy infinite dimensional) Hilbert spaces with $\operatorname{dim} H \leqslant \operatorname{dim} K$, then $\mathcal{L}(H, K)$ contains an isometry and products $A B^{*} C$ whenever it contains A, B and C. Thus, for example, Theorem 12 covers the case considered in Theorem 6.1 of [12].

Note that any product of upper half-planes of $\mathrm{J}^{*}$-algebras which each have an isometry is the upper half-plane of the product of the $\mathrm{J}^{*}$-algebras with respect to the product isometry.

Example 1. View the elements of $\mathcal{L}\left(C^{n}\right)$ as $n \times n$ matrices with complex entries and let $\mathscr{U}=\left\{A \in \mathscr{L}\left(\mathbf{C}^{n}\right): A^{t}=A\right\}$. Take $V=I$. Then $\mathscr{X}_{0}$ and $\mathscr{X}$ are Siegel's generalized unit disc and generalized upper half-plane, respectively. (See [35].) 
Example 2. Let $H$ be a Hilbert space and let $v$ be a unit vector in $H$. Then $v$ is an isometry since $v^{*} v=1$, and the corresponding upper half-plane is

$$
\pi=\left\{x \in H: \operatorname{Im}(x, v)>\|x-(x, y) v\|^{2}\right\}
$$

Example 3 (Fuks $[10, p .318]$ ). Let $\mathscr{U}=\mathcal{L}\left(\mathrm{C}^{n}, \mathrm{c}^{m}\right.$ ), where $\mathrm{n}<\mathrm{m}$ and view $\mathscr{\mathcal { C }}$ as the set of all $m \times n$ matrices with complex entries. Each $A \in \mathcal{U}$ can be written in the form $A=\left[\begin{array}{c}A_{1} \\ A_{2}\end{array}\right]$, where $A_{1}$ and $A_{2}$ are $n \times n$ and $(m-n) \times n$ matrices, respectively. Take $V=\left[\begin{array}{l}l \\ 0\end{array}\right]$, where $l$ is the identity $n \times n$ matrix. Then $V$ is an isometry and the corresponding upper half-plane is

$$
\mathscr{H}=\left\{\left[\mathrm{A}_{2} \mathrm{~A}_{2}\right]: \operatorname{Im} \mathrm{A}_{1}-\mathrm{A}_{2}^{*} \mathrm{~A}_{2}>0\right\} .
$$

Example 4 (cf. [3, p.149]). Let $H$ be a Hilbert space with conjugation $x \rightarrow \bar{x}$ and let $v$ be a unit vector in $H$ with $\bar{v}=v$. As shown in $\S 2$, there exists a linear transformation $x \rightarrow A_{x}$ satisfying (2) which allows us to identity $H$ with a Cartan factor $\mathscr{Y}$ of type IV. Take $V=A_{V}$ and note that $V$ is unitary. Since $\operatorname{Im} A_{V} A_{x}=$ $i\left[(\bar{x}, v) I-A_{v} A_{R e}\right]$ for all $x \in H$, it follows from (9) that the corresponding upper half-plane is

$$
\mathcal{H}=\{x \in H:(\operatorname{Im} x, v)>\|\operatorname{Re}[x-(x, v) v]\|\} .
$$

This domain is mapped by the invertible linear map $L x=(x, v) v+i[x-(x, v) v]$ onto the domain

$$
\tilde{F}=\{x \in H:(\operatorname{lm} x, v)>\|\operatorname{lm}[x-(x, v) v]\|\},
$$

which is a tube whose base is a future light cone. Also, $\tilde{S}=\operatorname{LoS}$ is a biholomorphic map of $\mathscr{U}_{0}$ onto $\widetilde{\mathscr{K}}$, and by (1) and (10).

$$
\widetilde{S}(x)=\frac{[1-(x, \bar{x})] v-2[x-[x, y) v]}{1-2(x, v)+(x, \bar{x})} .
$$

Proof of Theorem 13. Put $I M A=\operatorname{Im} V^{*} A-A^{*}\left(I-V V^{*}\right) A$ for $A \in \mathcal{U}$. It follows from Proposition 1 and the power series expansion for $S(A)$ that $S$ maps $\mathscr{2}_{0}$ into $\mathscr{U}$. By a computation,

$$
\left(M S(A)=\left(I-A^{*} V\right)^{-1}\left(I-A^{*} A\right)\left(I-V^{*} A\right)^{-1},\right.
$$

so $\mathrm{S}$ maps $\mathscr{\mathscr { U }}_{0}$ into $\mathcal{H}$. A similar identity (which can be derived from the one above) shows that the transformation $S^{-1}$ given by

$$
S^{-1}(A)=\left(A V^{*}+i\right)^{-1}(A-i V)
$$

maps $\mathcal{H}$ into $\mathscr{U}_{0}$. (Note that if $A \in \mathcal{H}$, the numerical range of $V^{*} A$ lies in the upper half-plane so $-i$ is not in the spectrum of $V^{*} A$.) It is easy to verify that $S^{-1}$ is the inverse of $S$ and that both $S$ and $S^{-1}$ are 
holomorphic where defined. Hence $S$ is a biholomorphic mapping of $\mathscr{Y}_{0}$ onto $\mathcal{H}$.

Clearly $\mathcal{H}$ is unbounded since it contains all positive multiples of $\mathrm{V}$. To see that $\mathcal{H}$ is convex, let $A, B \in \mathcal{H}$ and take $C=t A+(1-t) B$, where $0 \leqslant t \leqslant 1$. Then

$$
\begin{aligned}
I M C & >t A^{*}\left(I-V V^{*}\right) A+(1-t) B^{*}\left(I-V V^{*}\right) B-C^{*}\left(I-V V^{*}\right) C \\
& =t(1-t)(A-B)^{*}\left(I-V V^{*}\right)(A-B) \geqslant 0,
\end{aligned}
$$

so $C \in \mathcal{H}$

Suppose that $\mathscr{U}$ contains $A B{ }^{*} C$ whenever it contains $A, B$ and $C$. To show that $\mathcal{H}$ is affinely homogeneous, it suffices to show that for each $B \in \mathcal{H}$, the transformation

$$
\begin{aligned}
R_{B}(A)=V \operatorname{Re} V^{*} B & +V(I M B)^{1 / 2} V^{*} A(I M B)^{1 / 2} \\
& +i V B^{*}\left(I-V V^{*}\right)\left[2 A(I M B)^{1 / 2}+B\right] \\
& +\left(I-V V^{*}\right)\left[A(I M B)^{1 / 2}+B\right]
\end{aligned}
$$

is an invertible mapping of $\mathcal{H}$ onto itself with $R_{B}(i V)=B$. By hypothesis, the map $A \rightarrow A(I M \quad B)$ takes $\mathscr{I}$ into $\mathcal{Y}$, and since $(I M B)^{1 / 2}$ is the limit in the operator norm of a sequence of polynomials in $I M B$, it follows that the map $A \rightarrow A(I M B)^{1 / 2}$ takes $\mathcal{X}$ into $\mathscr{U}$. Hence $R_{B}$ maps $\mathcal{U}$ into $\mathscr{U}$. A computation shows that $R_{B}(i V)=B$ and that

$$
I M R_{B}(A)=(I M B)^{1 / 2}(I M A)(I M B)^{1 / 2} .
$$

Hence $R_{B}$ maps $\mathcal{F}$ into itself. It can be verified that the inverse of $R_{B}$ is given by

$$
R_{B}^{-1}(A)=V(I M B)^{-1 / 2}\left[V^{*} A-R e V^{*} B-i B^{*}\left(I-V V^{*}\right)(2 A-B)\right](I M B)^{-1 / 2}+\left(I-V V^{*}\right)(A-B)(I M B)^{-1 / 2}
$$

and that $R_{B}^{-1}$ maps $\mathscr{Y}$ into $\mathscr{U}$. It then follows from the identity given above that $R_{B}^{-1}$ maps $\mathscr{H}$ into itself. Therefore $R_{B}$ is as required.

Now suppose instead that $V$ is a unitary operator. Since the $J^{*}$-algebras $\mathscr{U}$ and $V^{*} \mathscr{U}$ are isometrically $J^{*}$-isomorphic, we may assume that $V=I$. Then by part (d) of Proposition 1 and the fact that $(\mathrm{Im} B)^{1 / 2}$ is the limit of a sequence of polynomials in Im B, the transformation

$$
R_{B}(A)=\operatorname{Re} B+(\operatorname{lm} B)^{1 / 2} A(\operatorname{lm} B)^{1 / 2}
$$

maps $\mathscr{U}$ into $\mathfrak{U}$. The argument given above now applies to show that $\mathfrak{H}$ is affinely homogeneous. 
Our construction and discussion of the domain $\mathcal{H}$ is based on the theory of Siegel domains of genus 2 given in [30, Ch. 1]. 


\section{Bibliography}

1. H. Behnke and P. Thullen, Theorie der Funktionen mehrerer komplexen Veränderlichen, 2nd edition, Ergebnisse der Math. 51, Springer, Berlin, 1970.

2. E. Berkson, Hermitian projections and orthogonality in Banach spaces, Proc. London Math. Soc. $24(1972), 101-118$.

3. É. Cartan, Sur les domaines bornés homogènes de l'espace de n variables complexes, Abh. Math. Sem. Univ. Hamburg 11(1935), 116-162.

4. , The Theory of Spinors, M.I.T. Press, Cambridge, Mass., 1966.

5. C. Chevalley, The Algebraic Theory of Spinors, Columbia Univ. Press, New York, 1954.

6. R. G. Douglas and D. Topping, Operators whose squares are zero, Rev. Romaine Math. Pures Appl. 12(1967), 647-652.

7. L. Druzkowski, Effective formula for the crossnorm in the complexified unitary spaces, (to appear).

8. N. Dunford and J. T. Schwartz, Linear Operators, part I, Interscience, New York, 1958.

9. C. J. Earle and R. S. Hamilton, A fixed point theorem for holomorphic mappings, Global Analysis, Proc. of Symposia in Pure Math. XVI, Amer. Math. Soc., Providence, R.I., 1965.

10. B. A. Fuks, Special Chapters in the Theory of Analytic Functions of Several Complex Variables, Transl. of Math. Monographs 14, Amer. Math. Soc., Providence, R.I., 1965.

11. S. Greenfield and N. Wallach, The Hilbert ball and bi-ball are holomorphically inequivalent, Bull. Amer. Math. Soc. 77(1971), 261-263.

12. Automorphism groups of bounded domains in Banach spaces, Trans. Amer. Math. Soc. $166(1972), 45-57$.

13. W. H. Greub, Multilinear Algebra, Grundlehren der math. Wissenshaften 136, Springer, New York, 1967.

14. L. A. Harris, Schwarz's lemma in normed linear spaces, Proc. Nat. Acad. Sci. U.S.A. 62(1969), $1014-1017$.

15. , Schwarz's lemma and the maximum principle in infinite dimensional spaces, thesis, Cornell University, Ithaca, N.Y., 1969 (available through University Microfilms, Inc., Ann Arbor, Michigan).

16. , A continuous form of Schwarz's lemma in normed linear spaces, Pacific J. Math. $38(1971), 635-639$.

17. , Banach algebras with involution and Möbius transformations, J. Functional Anal. $11(1972), 1-16$.

18. Bounds on the derivatives of holomorphic functions of vectors, (to appear in the proceedings of a conference held in Rio de Janeiro in 1972). 
19. L. K. Hua, Harmonic Analysis of Functions of Several Complex Variables in the Classical Domains, Transl. of Math. Monographs 6, Amer. Math. Soc., Providence, R.I., 1963.

20. R. V. Kadison, Isometries of operator algebras, Ann. of Math. 54(1951), 325-338.

21. R. V. Kadison and J. R. Ringrose, Derivations and automorphisms of operator algebras, Comm. Math. Phys. 4(1967), 32-63.

22. A. Korănyi and J. A. Wolf, Realization of hermitian symmetric spaces as generalized half-planes, Ann. of Math. 81(1965), 265-288.

23. K. Morita, Schwarz's lemma in a homogeneous space of higher dimensions, Japanese J. Math. $19(1944), 45-56$.

24. On the kernel functions for symmetric domains, Sci. Rep. Tokyo Kyoiku Daigaku, Sect. $A 5(1956), 190-212$.

25. M. A. Naimark, Normed Rings, P. Noordhoff, Groningen, Netherlands, 1964.

26. R. Narasimhan, Several Complex Variables, University of Chicago Press, Chicago, 1971.

27. E. Peschl and F. Erwe, Uber beschränkte Systeme von Funktionen, Math. Ann. 126(1953), $185-220$.

28. R. S. Phillips, On symplectic mappings of contraction operators, Studia Math. 31(1968), $15-27$.

29. V.P. Potapov, The multiplicative structure of $\mathbf{J}$-contractive matrix functions, Amer. Math. Soc. Transi. $15(1960), 131-243$.

30. Pyatetskii-Shapiro, Automorphic Functions and the Geometry of Classical Domains, Gordon and Breach, New York, 1969.

31. C. E. Rickart, General Theory of Banach Algebras, Van Nostrand, Princeton, N.J., 1960.

32. B. Russo and H. A. Dye, A note on unitary operators in C*-algebras, Duke Math. J. 33(1966), $413-416$.

33. S. Sakai, $C^{*}$-algebras and $W^{*}$-algebras, Ergebnisse der Math. 60, Springer, Berlin, 1971.

34. I. E. Segal, Tensor algebras over Hilbert spaces II, Ann. of Matii. 63(1956), 160-175.

35. C. L. Siegel, Symplectic geometry, Amer. J. Math. 65(1943), 1-86.

36. Analytic Functions of Several Complex Variables, Lecture notes at the Institute for Advanced Study, Princeton, N.J., 1948-1949.

37. E. Thorp and R. Whitley, The strong maximum modulus theorem for analytic functions into a Banach space, Proc. Amer. Math. Soc. 18(1967), 640-646.

38. D. Topping, An isomorphism invariant for spin factors, J. Math. Mech. 15(1966), 1055-1063.

Department of Mathematics

University of Kentucky

Lexington, Kentucky 40506, USA 